\title{
Experimental contamination of Minims of fluorescein by Pseudomonas aeruginosa
}

\author{
CHARLES CLAOUÉ \\ From the University of Bristol Department of Ophthalmology, Bristol Eye Hospital, Lower Maudlin Street, \\ Bristol BS1 2LY
}

Summary Contamination of fluorescein solutions by Pseudomonas aeruginosa has been a concern of ophthalmologists for many years because of the severity of pseudomonas keratitis. Attempts to prevent contamination have been directed at stringent sterility control during manufacture and the introduction of single-dose disposable containers such as Minims. Deliberate contamination of Minims fluorescein with Pseudomonas aeruginosa was attempted. Under conditions likely to be met with in clinical practice the contents remained sterile. However, under extreme conditions of immersion in pure broth culture of Pseudomonas aeruginosa contamination could be achieved. The relevance of these results to clinical practice is discussed.

Contamination of fluorescein solutions by Pseudomonas aeruginosa has long caused concern ${ }^{2}$ because of the severity of pseudomonas keratitis. ${ }^{3}$ Fluorescein solution is widely used to delineate corneal epithelial deficits, ${ }^{4}$ particularly in casualty departments, where superficial anterior segment trauma is commonly seen. ${ }^{56}$ The corneal epithelium is a natural anatomical barrier to infection, and defects whether due to trauma (abrasions), virus infection (dendritic ulcers), or degeneration (ruptured bullae) are portals to the stroma for bacterial pathogens.

Although the commonest cause of a central corneal ulcer is herpes simplex, ${ }^{7}$ bacterial corneal ulcers still occur frequently, and, of these, Pseudomonas aeruginosa keratitis is the most common. ${ }^{3}$ Since fluorescein solutions are known to be capable of contamination with Ps. aeruginosa, ${ }^{12}$ considerable effort has been spent on minimising the risk of an already compromised cornea receiving an inoculum of pseudomonas when fluorescein is applied. One result has been the introduction of single-dose disposable containers of fluorescein such as Minims and others. While reputable and conscientious pharmaceutical companies will ensure that the fluorescein is sterile when dispatched from the point of manufacture, the risk of accidental contamination at the point of use does not appear to have been previously assessed.

Correspondence to Dr C. Claoué, 36a Queens Gardens, Bayswater, London W2.

\section{Materials and methods}

Fluorescein Minims consist of 1 or $2 \%$ preservativefree fluorescein in a polypropylene Minims bottle which tapers to a neck. The neck and exit pore are covered by a polypropylene cap. A paper and polypropylene overwrap enclose the bottle and cap.

Experiment 1. One hundred Minims of $2 \%$ fluorescein were removed from their overwraps and immersed in a broth culture of $5.5 \times 10^{\times}$colony forming units (cfu) per ml of a recently isolated strain of Pseudomonas aeruginosa. After 5 minutes the bottles were removed, shaken dry, the caps carefully removed, and a single drop of the fluorescein plated on to nutrient agar. Great care was taken to ensure that no drop of broth on the bottle was allowed to run up the neck after this had been exposed. The agar plates were cultured at $37^{\circ} \mathrm{C}$ for 24 hours, at which time any growth of pseudomonas was considered to represent contamination of the fluorescein. As with subsequent experiments, appropriate control plates were used to check for aerosol contamination of culture plates.

Experiment 2. One hundred Minims of $2 \%$ fluorescein in overwraps were immersed in a broth culture of $9.5 \times 10^{\text {h }} \mathrm{cfu}$ per $\mathrm{ml}$ of a recently isolated strain of $P S$. aeruginosa. After 5 minutes they were removed and shaken dry. Each overwrap was opened, the Minims 
bottle removed, and a single drop of the fluorescein plated on to nutrient agar, which was incubated as in experiment 1.

Experiment 3. One hundred Minims of $2 \%$ fluorescein were subdivided into two equal groups, one of which had the overwraps removed. With surgical gloves worn the hands were contaminated by immersion in a broth culture of $8.5 \times 10^{5} \mathrm{cfu}$ per $\mathrm{ml}$ of a recently isolated strain of Ps. aeruginosa and then lightly dried. A single drop of fluorescein was then plated on to nutrient agar from each Minims bottle, the overwraps being removed where necessary. After every 10 operations the gloves were discarded, cultured, and a new pair contaminated in an identical fashion before proceeding. Incubations were as in experiment 1 .

\section{Results}

The results are presented in Table 1 . It can be seen that direct immersion of Minims bottles (experiment 1) in broth culture of Pseudomonas aeruginosa resulted in contamination of the vast majority, but that the presence of the paper and polypropylene overwrap in experiment 2 reduced this to only a few contaminated. When the bottles were manipulated with gloves contaminated with Ps aeruginosa (all of which were culture proved) the rate of contamination was reduced yet further irrespective of whether the overwrap had been previously removed.

\section{Discussion}

When Minims bottles of fluorescein are removed from their overwraps and immersed in high titre broth cultures of Pseudomonas aeruginosa, the first drop of fluorescein used is contaminated in the overwhelming majority of cases. It seems unlikely that the organism would be able to penetrate the relatively thick polypropylene walls of the Minims bottle. Since care was taken to ensure that drops of broth did not run down the side of the bottle and

Table 1 Number of plates of nutrient agar which grew Pseudomonas aeruginosa after 24 hours' incubation at $37^{\circ} \mathrm{C}$. In experiment $3 a$ the overwraps were removed prior to manipulation by contaminated gloves, whereas in experiment $3 b$ they were removed by the infected gloves

\begin{tabular}{ll}
\hline Experiment & $\begin{array}{l}\text { Number of plates which grew } \\
\text { Pseudomonas aeruginosa }\end{array}$ \\
\hline 1 & 99 \\
2 & 3 \\
3a & 0 \\
3b & 0 \\
\hline
\end{tabular}

along the neck, this can also be discounted as the cause of the contamination. It is more probable that a meniscus of pseudomonas-contaminated broth was formed at the base of the neck adjacent to the opening of the cap. When the cap was removed, the meniscus would be smeared along the neck to the exit pore. This mechanism for contamination of eye drops has previously been described. ${ }^{89}$ Preliminary experiments (data not shown) suggest that, if the broth is painted on with a bacteriological loop rather than immersion of the bottle, then the contamination rate is reduced to the very low levels seen in experiment 3 . This provides further support for the suggested mechanism of contamination.

The presence of an intact overwrap significantly reduces the incidence of contamination after immersion in broth culture. Contamination probably occurs when the pseudomonas broth leaks through the paper section of the overwrap, producing a situation identical to that found in experiment 1 . When contamination was attempted by using infected hands (gloves) to manipulate the Minims bottles, the level of contamination of the fluorescein was reduced to a very low level irrespective of whether the overwrap was previously removed or not.

Whereas contamination of the fluorescein could regularly be obtained by immersing Minims bottles in a high titre broth of pseudomonas, it seems inconceivable that such a situation would arise in clinical practice. Furthermore, the inoculum required to produce disease in the human eye, and the size and frequency of such inocula are unknown. A recent study in hospital staff has shown that Ps. aeruginosa is capable of colonising the skin under wedding rings," thus creating a situation analogous to experiment 3 . Since no contamination of fluorescein was produced by manipulation with experimentally contaminated gloves, it may be concluded that this potential reservoir of infection is not a significant risk to patients.

This study was funded in part by the Wellcome Trust. I should like to thank Dr Graham Hopkins of Smith and Nephew Pharmaceuticals Ltd for helpful discussions and supplies of Minims. The technical assistance of the following has been invaluable: Mrs Christine Harber, Mr Tim Hodges, and Mr Paul Strickland. Mr Andrew Elkington, FRCS, and Miss Katherine Stevenson, FRCS, contributed helpful discussions, and I should like to thank Dr Bill Blyth and Professor David Easty, FRCS, for reading the manuscript.

Minims is a trade mark of Smith and Nephew Pharmaceuticals Ltd.

\section{References}

1 McCulloch JC. Origin and pathogenicity of Ps. pyocyanea in the conjunctival sac. Arch Ophthalmol 1943; 29: 924-36.

2 Vaughan DG. Contamination of fluorescein solutions. Am J Ophthalmol 1955; 39: 55-61.

3 Smolin G. Tabbara K. Whitcher J. Infectious diseases of the eye. Baltimorc: Williams and Wilkins, 1984:86-7. 
4 Newell FW. Ophthalmology, principles and concepts. St Louis: Mosby, 1982:148.

5 Vernon SA. Analysis of all new cases seen in a busy regional centre ophthalmic casualty department during a 24 weeks period. $J R$ Soc Med 1983; 76: 279-82.

6 Jones NP, Hayward MJ, Khaw PT, Claouć CMP. Elkington AR. An analysis of the function of an ophthalmic accident and emergency department-a six month survey. Br Med $J$ in press.

7 Yanoff M. Fine BS. Cornea and sclera. In: Duane TD. Jacger EA, cds. Biomedical foundations of ophthalmology. Philadelphia: Harper and Row, 1983: 3: 10.
8 Templeton WC, Eiferman RA. Snyder IW. Melo JC. Raff MJ. Serratia keratitis transmitted by contaminated eyedroppers. Am J Ophthalmol 1982: 93: 723-6.

9 Coad CT, Osato MS. Wilhelmus KR. Bacterial contamination of eyedrop dispensers. A in J Ophthalmol 1984: 98: 548-51.

10 Hoffman PN. Cooke EM. McCarville MR. Emmerson AM. Micro-organisms isolated from wedding rings worn by hospital staff. $B r$ Med J 1985; 290: 206-7.

Accepted for publication 29 November 1985. 